\title{
openheart Evaluation of the prognostic value of GDF-15, ABC-AF-bleeding score and ABC-AF-death score in patients with atrial fibrillation across different geographical areas
}

\author{
Tymon Pol (10 ,1,2 Ziad Hijazi, ${ }^{1,2}$ Johan Lindbäck (D) ,2 John H Alexander, ${ }^{3}$ \\ M Cecilia Bahit, ${ }^{4}$ Raffaele De Caterina (D) , ${ }^{5} \mathrm{JW}$ Eikelboom, ${ }^{6}$ Michael D Ezekowitz, ${ }^{7}$ \\ Bernard J Gersh, ${ }^{8}$ Christopher B Granger, ${ }^{3}$ Elaine M Hylek, ${ }^{9}$ Renato Lopes, ${ }^{3}$ \\ Agneta Siegbahn, ${ }^{2,10}$ Lars Wallentin ${ }^{1,2}$
}

\begin{abstract}
- Additional material is published online only. To view, please visit the journal online (http://dx.doi.org/10.1136/ openhrt-2020-001471)
\end{abstract}

To cite: Pol T, Hijazi Z, Lindbäck J, et al. Evaluation of the prognostic value of GDF-15, ABC-AF-bleeding score and

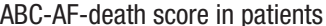
with atrial fibrillation across different geographical areas. Open Heart 2021;8:e001471. doi:10.1136/

openhrt-2020-001471

Received 15 0ctober 2020 Revised 26 January 2021 Accepted 6 March 2021
Check for updates

(c) Author(s) (or their employer(s)) 2021. Re-use permitted under CC BY. Published by BMJ.

For numbered affiliations see end of article.

Correspondence to Dr Tymon Pol; tymon.pol@ medsci.uu.se

\section{ABSTRACT}

Objectives Growth differentiation factor 15 (GDF-15) is a biomarker independently associated with bleeding and death in anticoagulated patients with atrial fibrillation (AF). GDF-15 is also used as one component in the more precise biomarker-based $A B C$ (age, biomarkers, clinical history)-AF-bleeding and ABC-AF-death risk scores. Data from large trials indicate a geographic variability in regard to overall outcomes, including bleeding and mortality risk. Our aim was to assess the consistency of the association between GDF-15, ABC-AF-bleeding score and ABC-AFdeath score, with major bleeding and death, across world geographic regions.

Methods Data were available from 14767 patients with AF from the Apixaban for Reduction in Stroke and Other Thromboembolic Events in Atrial Fibrillation (ARISTOTLE) trial and 8651 patients with $\mathrm{AF}$ from the Randomized Evaluation of Long-Term Anticoagulation Therapy (RELY) trial in this cohort study. GDF-15 was analysed from plasma samples obtained at randomisation. The geographical consistency of the associations between outcomes and GDF-15, ABC-AF-bleeding score and ABCAF-death scores were assessed by Cox-regression models including interactions with predefined geographical region. Results GDF-15 and the ABC-AF-bleeding score were associated with major bleeding in both trials across regions $(p<0.0001)$. Similarly, GDF-15 and the ABC-AFdeath score were associated with all-cause mortality in both trials across regions $(p<0.0001)$. Overall, the association between GDF-15, the ABC-AF-bleeding score and $\mathrm{ABC}-\mathrm{AF}$-death risk score with major bleeding and death was consistent across regions in both ARISTOTLE and the RE-LY trial cohorts. The ABC-AF-bleeding and $A B C-A F-d e a t h$ risk scores were consistent regarding discriminative ability when comparing geographic regions in both trial cohorts. The $\mathrm{C}$-indices ranged from 0.649 to 0.760 for the ABC-AF-bleeding and from 0.677 to 0.806 for the ABC-AF-death score by different geographic regions.

Conclusions In patients with $\mathrm{AF}$ on anticoagulation, GDF-15 and the biomarker-based ABC-AF-bleeding and

\section{Key questions}

What is already known about this subject?

- Growth differentiation factor 15 (GDF-15) and the biomarker-based $\mathrm{ABC}-\mathrm{AF}$-bleeding and $\mathrm{ABC}-\mathrm{AF}$ death risk scores are independently associated with bleeding and death in anticoagulated patients with atrial fibrillation (AF). Their prognostic value across geographical regions has however not been evaluated previously.

What does this study add?

- This study shows that GDF-15 and the ABC-AFbleeding and $\mathrm{ABC}-\mathrm{AF}$-death risk scores have similar prognostic value across world geographic regions.

How might this impact on clinical practice?

- The findings from this study add novel information regarding the consistency of GDF-15 and the $\mathrm{ABC}$-AF-risk scores in $\mathrm{AF}$ and strengthen their role as a risk refinement tool applicable for a wide international usage. Furthermore, the assessment of geographic variation might be helpful as part of a validation process when introducing a new biomarker or risk score intended for a wide use.

ABC-AF-death risk scores are consistently associated with respectively increased risk of major bleeding and death and have similar prognostic value across world geographic regions.

Trial registration number ClinicalTrials.gov Registry NCT00412984 and NCT00262600.

\section{INTRODUCTION}

In patients with atrial fibrillation $(\mathrm{AF})$ an individual risk assessment is recommended. ${ }^{1}$ Several biomarkers have recently been shown to add independent prognostic value regarding the risk of bleeding and death 
in $\mathrm{AF}^{2-5}$ Growth differentiation factor 15 (GDF-15) is a marker of oxidative stress and inflammation and has been demonstrated to be strongly associated with major bleeding and death, but less with stroke, in patients with AF. ${ }^{6}$ These properties make GDF-15 an attractive candidate in the search for markers that facilitate the distinction between the risk of stroke against the risk of bleeding. GDF-15 has furthermore been incorporated into the two clinical risk scores in AF, the ABC-AFbleeding and the ABC-AF-death scores (age, biomarkers and clinical history), providing improved risk assessment concerning these outcomes in $\mathrm{AF}$ and outperforming other risk scores in this scenario. ${ }^{89}$ However, recent data from large Non-Vitamin K Antagonist Oral Anticoagulants) trials have indicated a geographic variability of cardiovascular outcomes, including bleeding risk and mortality risk, even after adjustment for clinical factors and biomarkers. ${ }^{10-14}$ At present, it is unknown whether the risks associated with GDF-15 for major bleeding and death are similar across geographic regions, as well as the performance of the ABC-AF-bleeding and ABC-AF-death scores, in which GDF-15 is incorporated. Therefore, in this study, we aimed to evaluate GDF-15 and the ABC-AFbleeding and ABC-AF-death scores across geographical regions using data from both the Apixaban for Reduction in Stroke and Other Thromboembolic Events in Atrial Fibrillation (ARISTOTLE) trial and the Randomized Evaluation of Long-Term Anticoagulation Therapy (RELY) trial.

\section{METHODS}

\section{Study population and trial design}

The details and outcomes of the ARISTOTLE and RE-LY trials have been described and published previously. ${ }^{15-18}$ The ARISTOTLE trial randomised 18201 patients from 39 countries with $\mathrm{AF}$ and at least one additional risk factor for stroke or systemic embolism to either warfarin or apixaban. The participating countries were categorised into prespecified regions: Asia/Pacific, Europe, Latin America and North America (online supplemental figure 1). This biomarker substudy used data from 14949 patients with plasma collected at randomisation, out of which 14688 had all biomarkers available, with a median follow-up of 1.8 (IQR: 1.3, 2.3) years. In the RE-LY trial, 18113 patients with $\mathrm{AF}$ were randomly assigned to dabigatran or warfarin. In total, patients from 44 countries were included in this trial. These countries were divided according to geographic regions (Asia, Europe, Latin America, North America and other) (online supplemental figure 1). A total of 9369 patients had biomarker data available from samples collected at randomisation, out of which 8402 had all biomarkers available, with a median follow-up time of 2.0 (IQR: 1.7, 2.3) years.

In both trials, all-cause mortality was a prespecified outcome and major bleeding was the primary safety outcome. ${ }^{1518}$ Major bleeding was in both trials defined according to the criteria of the International Society on
Thrombosis and Haemostasis. ${ }^{15} 18$ Blinded clinical events committees reviewed and centrally adjudicated these outcome events.

\section{The ABC-AF-bleeding and ABC-AF-death scores}

As previously described, the ABC-AF scores for prognostication of outcomes were developed with the objective to include a minimal number of the strongest markers among the candidate variables: $\mathrm{A}=\mathrm{Age}, \mathrm{B}=$ Biomarkers and $\mathrm{C}=$ Clinical factors. ${ }^{8}{ }^{9}$ The ABC-AF- bleeding score therefore consists of age, the levels of haemoglobin, high-sensitive cardiac troponin T (cTnT-hs) and GDF-15 and finally previous bleeding as the only necessary clinical factor. The ABC-AF-death score was developed in a similar way and incorporates age, the biomarkers N-terminal pro B-type natriuretic peptide (NT-proBNP), cTnT-hs, GDF-15 and a clinical history of heart failure.

\section{Laboratory methods}

Venous blood samples were obtained at randomisation from enrolled patients in both trials. The samples were centrifuged and plasma stored in aliquots and subsequently transferred to the Uppsala Clinical Research Centre (UCR) laboratory, Uppsala, Sweden, for storage at $-70^{\circ} \mathrm{C}$ and central analyses. ${ }^{15}$ GDF-15 was analysed with the Elecsys GDF-15 precommercial assay kit P03 (Roche Diagnostics, Mannheim, Germany). The assay is reported to have an interassay coefficient of variation of $2.3 \%$ at $100 \mathrm{ng} / \mathrm{L}$ and $1.8 \%$ at $17200 \mathrm{ng} / \mathrm{L}$; the intra-assay coefficient of variation was $0.8 \%$ at $1100 \mathrm{ng} / \mathrm{L}$ and $0.9 \%$ at $18600 \mathrm{ng} / \mathrm{L}$ with a lower detection limit $<10 \mathrm{ng} / \mathrm{L}$. When intra-assay and interassay variabilities were combined, the coefficients of variation at our laboratory were $4.4 \%$ at $1500 \mathrm{ng} / \mathrm{L}$ and $4.5 \%$ at $5900 \mathrm{ng} / \mathrm{L}$. ${ }^{6}$ The details for the analyses of the other biomarkers in the ABC-AF-bleeding and ABC-AF-death scores have previously been published, in short, cardiac troponin-T (cTnT-hs) and NT-proBNP levels were analysed with high-sensitivity immunoassays on the Cobas Analytics e601 (Roche Diagnostics, Mannheim, Germany). ${ }^{8}$ All analyses were done according to the instructions of the manufacturer.

Data collected in the ARISTOTLE and RE-LY trials (case report form, outcomes, laboratory samples and analyses) were standardised and congruent in both individual trials for all participating. GDF-15 analyses were in both trials analysed centrally in the UCR laboratory. Specific information regarding data collection and handling can be found in the original trial publications. ${ }^{15-18}$

\section{Statistical analysis}

To assess the consistency over geographic regions regarding the association between time to major bleeding (or time to death) and GDF-15, a Cox-regression model including GDF-15, geographic region and their interaction was fitted. To assess the consistency of the ABC-AF scores, a corresponding model was fitted including the ABC-AF-bleeding (ABC-AF-death) score, here represented by the estimated 1-year risk for major bleeding 
(death), instead of GDF-15. In all models, GDF-15 and the ABC-AF scores were log-transformed (natural logarithm) and represented as restricted cubic splines with four knots placed at the 5th, 35th, 65th and 95th sample percentiles of the corresponding variable. The interaction term only included the linear part of GDF-15 or the ABC-AF score, respectively. Thus, the test of the hypothesis of no interaction between geographic region and GDF-15 (or the ABC-AF score) is the test for the regression coefficient of region $\times \ln (\mathrm{GDF}-15)$ (or region $\times$ the ABC-AF score) being zero in the model.

The associations are illustrated graphically by plotting the estimated relative hazard, with corresponding $95 \%$ confidence bands. For the models including GDF-15, an arbitrary reference was set at a GDF-15 value of 1500 $\mathrm{ng} / \mathrm{L}$ for someone in Europe. For the ABC-AF scores, the reference point was set to an arbitrary value of 2\% 1-year risk, also for someone in Europe. The horizontal range of the curves approximately represents the range of GDF-15 (ABC-AF score) after removal of the 10 lowest and 10 highest values.

Harrell's c-index was used to assess the discriminative ability of GDF-15 and the ABC-AF scores, both overall and within regions.

All analyses were done in R, V.3.5.2, ${ }^{19}$ using the $\mathrm{rms}^{20}$ add-on package.

\section{RESULTS \\ Demographic data}

In total, 14688 patients from the biomarker cohort of the ARISTOTLE trial as well as 8402 patients from the RE-LY trial had all biomarker concentrations available at randomisation. The number of patients and events in each trial as well as baseline demographics and biomarker levels according to the prespecified geographic regions of both

Table 1 Baseline characteristics of the 14949 patients in the ARISTOTLE cohort according to study region

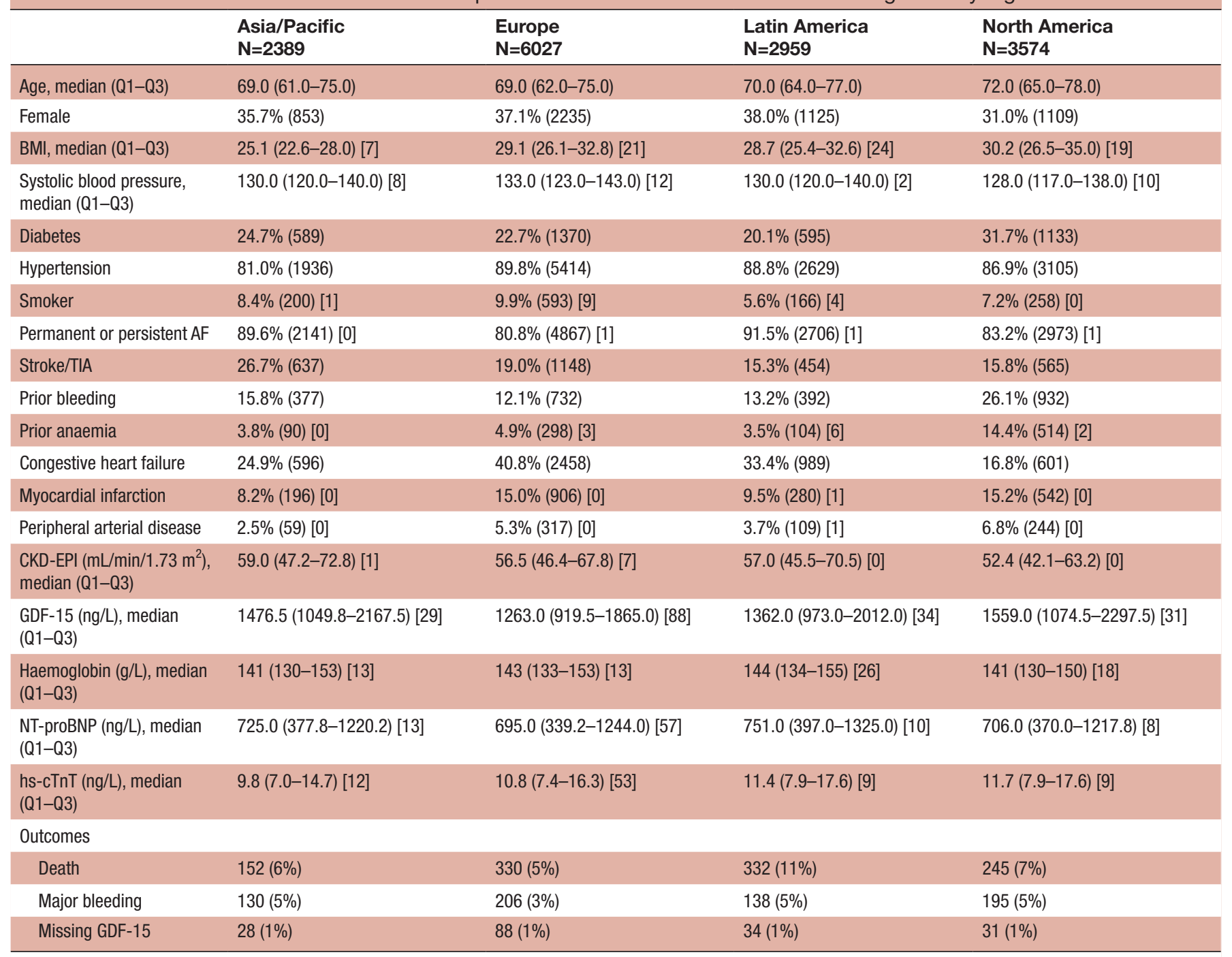

Number in brackets represents number of missings.

AF, atrial fibrillation; ARISTOTL, Apixaban for Reduction in Stroke and Other Thromboembolic Events in Atrial Fibrillation; BMI, body mass index; CKD-EPI, Chronic Kidney Disease Epidemiology Collaboration equation; GDF-15, growth differentiation factor 15; hs-cTnT, high-sensitive cardiac troponin T; NT-proBNP, N-terminal pro B-type natriuretic peptide; Q, quartile; TIA, transient ischaemic attack. 
trials are shown in tables 1 and 2. All participating countries in both trial cohorts are shown in online supplemental figure 1 . The median age in the ARISTOTLE trial cohort was 70 (IQR: 63,76 ) years and $64 \%$ were men, while in the RE-LY cohort, the median age was 72 (IQR: 67, 77) years with the same proportion of men, $64 \%$. The largest proportion of patients was from Europe in both trial cohorts, $40 \%$ in ARISTOTLE and 54\% in RE-LY.

\section{Association of GDF-15 with major bleeding by geographic region}

In the ARISTOTLE biomarker cohort, GDF-15 was associated with major bleeding across all the study geographic regions $(\mathrm{p}<0.0001)$. The risk of major bleeding differed among regions $(p=0.02)$ but there was no statistically significant interaction between GDF-15 and specific regions $(p=0.80)$. The associations between GDF-15 and major bleeding by different regions are shown in figure 1 (top left panel).

In the RE-LY biomarker cohort, the results were similar, as GDF-15 was associated with major bleeding $(p<0.0001)$. The risk of bleeding varied among regions $(\mathrm{p}<0.0001)$ as was the case in the ARISTOTLE biomarker cohort, the interaction between GDF-15 and specific regions was not statistically significant $(p=0.08)$. The associations between GDF-15 and major bleeding by regions in the RE-LY biomarker cohort are shown in figure 1 (top right panel).

\section{Association of GDF-15 with death by geographic region}

GDF-15 was associated with death in the ARISTOTLE biomarker cohort $(p<0.0001)$. The risk of death differed among regions $(p<0.0001)$ but the interaction between GDF-15 and regions was not statistically significant $(\mathrm{p}=0.12)$. The associations between GDF-15 with death by regions are shown in figure 1 (bottom left panel).

In the RE-LY biomarker cohort, the results were similar (figure 1, bottom right panels), with a strong association between GDF-15 and death $(\mathrm{p}<0.0001)$. Similarly, the mortality risk differed between regions $(\mathrm{p}=0.005)$ and the interaction between GDF-15 and regions was not statistically significant $(\mathrm{p}=0.84)$.

\section{Geographic evaluation of the ABC-AF-bleeding risk score}

In the ARISTOTLE biomarker cohort, the ABC-AFbleeding score was associated with major bleeding across all regions $(\mathrm{p}=0.0001)$ without a statistically significant interaction between the ABC-AF-bleeding score and regions $(\mathrm{p}=0.91)$. The associations between the ABC-AFbleeding score with major bleeding by regions are shown in figure 2 (top left panel).

In the RE-LY biomarker cohort, the results were similar (figure 2, top right panel), as the ABC-AF-bleeding risk score was associated with major bleeding across geographic regions $(\mathrm{p}=0.0001)$ without a statistically significant interaction between the ABC-AF-bleeding score and regions $(\mathrm{p}=0.11)$.
Comparison of the discrimination of the ABC-AFbleeding risk score showed consistency across all studied geographic regions in both the ARISTOTLE and RE-LY trial cohorts (table 3 ).

\section{Geographic evaluation of the ABC-AF-death risk score}

In both the ARISTOTLE and RE-LY biomarker cohorts, the ABC-AF-death score was associated with all-cause mortality across all regions $(\mathrm{p}=0.0001)$ (figure 2 , bottom panels). However, in both cohorts, we observed a higher mortality at lower ABC-AF-death scores in Latin America than in the other regions leading to a significant quantitative interaction with region in the ARISTOTLE cohort (interaction- $\mathrm{p}=0.018$ ) although not in the RE-LY cohort ( $\mathrm{p}=0.365)$ (figure 2, bottom panels).

The discrimination of the ABC-AF-death risk score was similar among geographic regions in both trial cohorts (table 4).

\section{DISCUSSION}

The major findings of this study were that GDF-15 and the ABC-AF-bleeding and ABC-AF-death scores were consistently associated with major bleeding and mortality across all studied geographic regions. There was no significant interaction between GDF-15 in regard to outcomes by regions in either trial cohort. Results were similar for the ABC-AF-risk scores although there seemed to be a quantitative interaction between ABC-AF-death score and region with a higher mortality at lower ABC-AF-death scores in Latin America than other regions. The ABC-AFbleeding and ABC-AF-death risk scores were consistent regarding their discriminative ability when comparing geographic regions in both the ARISTOTLE and the RE-LY trial cohorts.

Among the various biomarkers and risk scores that have been explored in regard to AF and its complications, none have to our knowledge been systematically examined regarding risk across geographic regions. Geographic differences could affect the predictive ability of these biomarkers and risk scores, making them less reliable in certain regions. This could be attributable to a number of factors, including genetic variation and regional treatment differences. When compared with other regions, for example, AF patients from Asia have both a higher risk of ischaemic stroke and bleeding. ${ }^{10-13}$ It is unknown how this increased risk in a specific region interacts with the risk associated with biomarkers and risk scores in AF. The assessment of geographic variation might therefore be helpful as part of a validation process when introducing a new biomarker or risk score intended for a wide use.

In the present study, there was a strong association between GDF-15, the ABC-AF-bleeding score and ABC-AFdeath risk score with major bleeding and death independent of geographic regions and countries. The slope in figures 1 and 2 appears steeper for the ABC-AF- scores as compared with GDF-15 possibly implying a better discriminatory and 


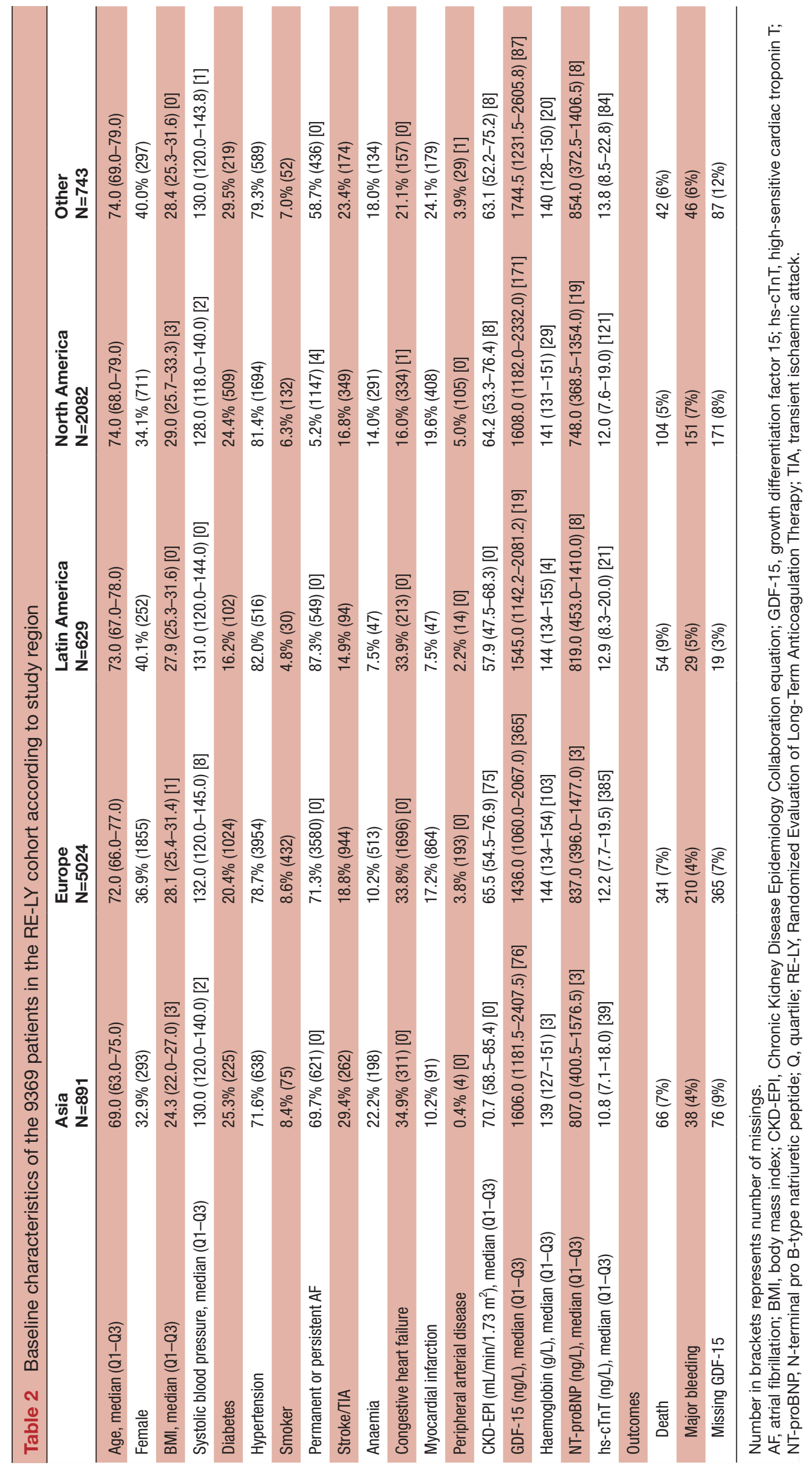



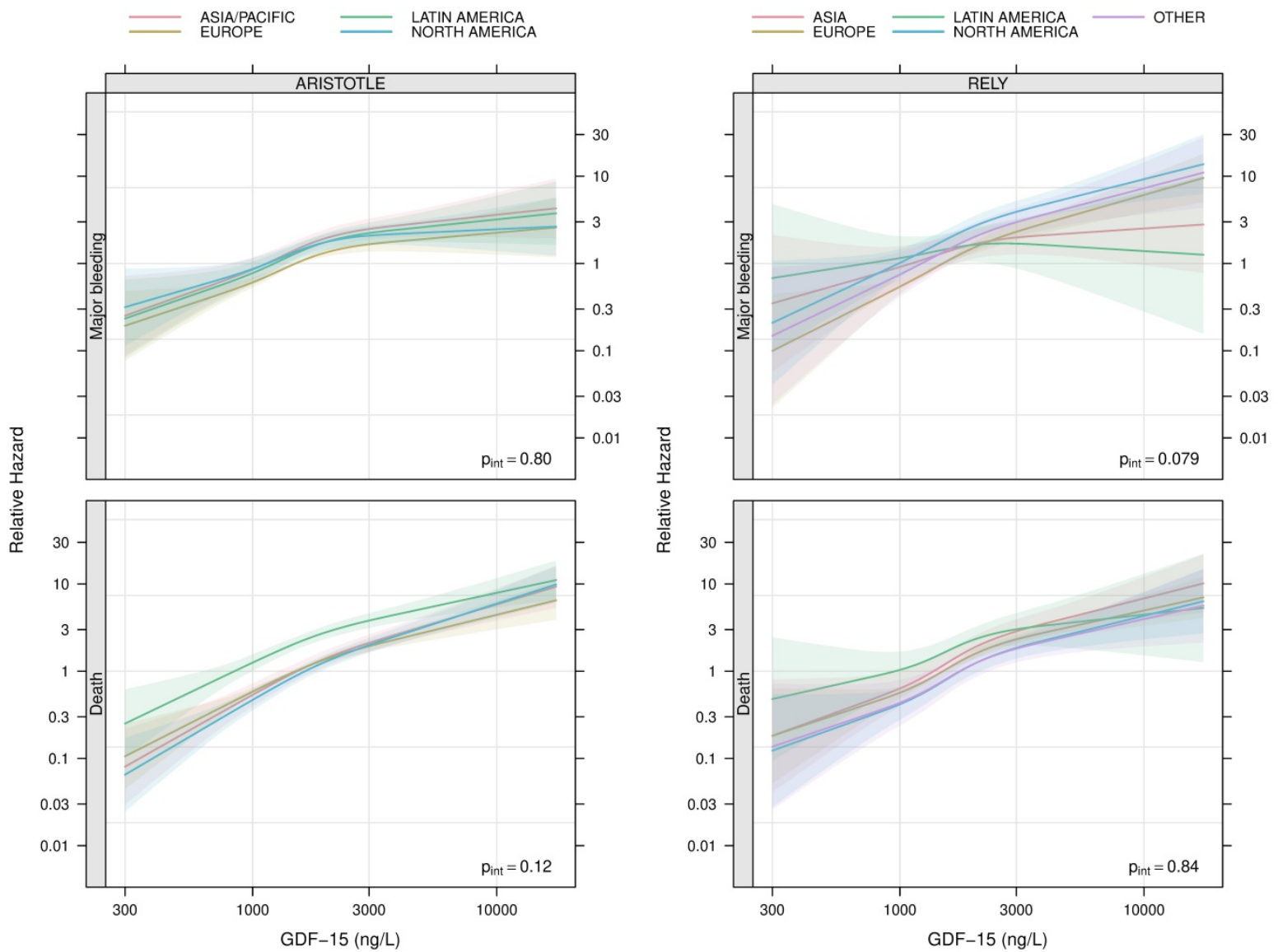

Figure 1 Relative hazard of major bleeding (top panels) and death (bottom panels) in relation to levels of GDF-15 among patients from different regions in the ARISTOTLE (left panels) and RE-LY (right panels) trials. An arbitrary reference point is set at a GDF-15 value of $1500 \mathrm{ng} / \mathrm{L}$ in Europe. The $p$ value in each panel is for a test of no interaction between region and GDF15. ARISTOTLE, Apixaban for Reduction in Stroke and Other Thromboembolic Events in Atrial Fibrillation; GDF-15, growth differentiation factor 15; RE-LY, Randomized Evaluation of Long-Term Anticoagulation Therapy.

risk assessment power of the ABC-AF- risk scores that most likely can be attributed the ABC-AF scores also including other predictive variables. There was a statistically significant interaction between the ABC-AF-death score and allcause mortality for geographic regions in the ARISTOTLE cohort. This interaction could be explained by a somewhat weaker association between the ABC-AF-death score and death in Latin America where patients had a generally higher mortality risk as compared with the other regions, especially for those having a low ABC-AF-death score. Although the interaction was not statistically significant, a similar pattern was seen in the RE-LY cohort suggesting a possibly weaker association between the ABC-AF-death score and mortality in Latin America compared with the other regions. However, as the discriminatory ability of the ABC-AF-death score was consistent across geographic regions, this eventual interaction is likely to be less relevant from a clinical perspective. Otherwise, the variability between the regions concerning the association with the outcomes for GDF-15 and the ABC-AF scores, that is, the slopes of the lines in figures 1 and 2, were almost negligible in comparison with the strong associations with outcomes within the regions.
The findings from this study therefore indicate an overall consistency of the performance of GDF-15 and the ABC-AF-risk scores across geographic regions.

Although this is the first study to systematically explore geographic variation concerning risk association of GDF-15 and the ABC-AF-risk scores, some limitations and strengths may be worth mentioning. The results are based on post hoc analyses, and it is well known that lowering the number of events by creating subgroups reduces the statistical power to detect true differences. In order to increase the certainty of the results and to minimise the risk of random findings, we used data from two cohorts as any inconsistency appearing in both trials would lower the risk of it being a chance finding. Even though a large number of patients from a variety of countries were represented in each region, the results of these analyses are limited to the participating countries. Further, the large sample size including two different trial cohorts with a global patient representation of contemporary data, using similar variable definitions and outcomes, are some of the major strengths of this study. Despite convincing documentation, the ABC-AF scores currently are not routinely used. Part of that may be due to additional time and cost to obtain biomarker 

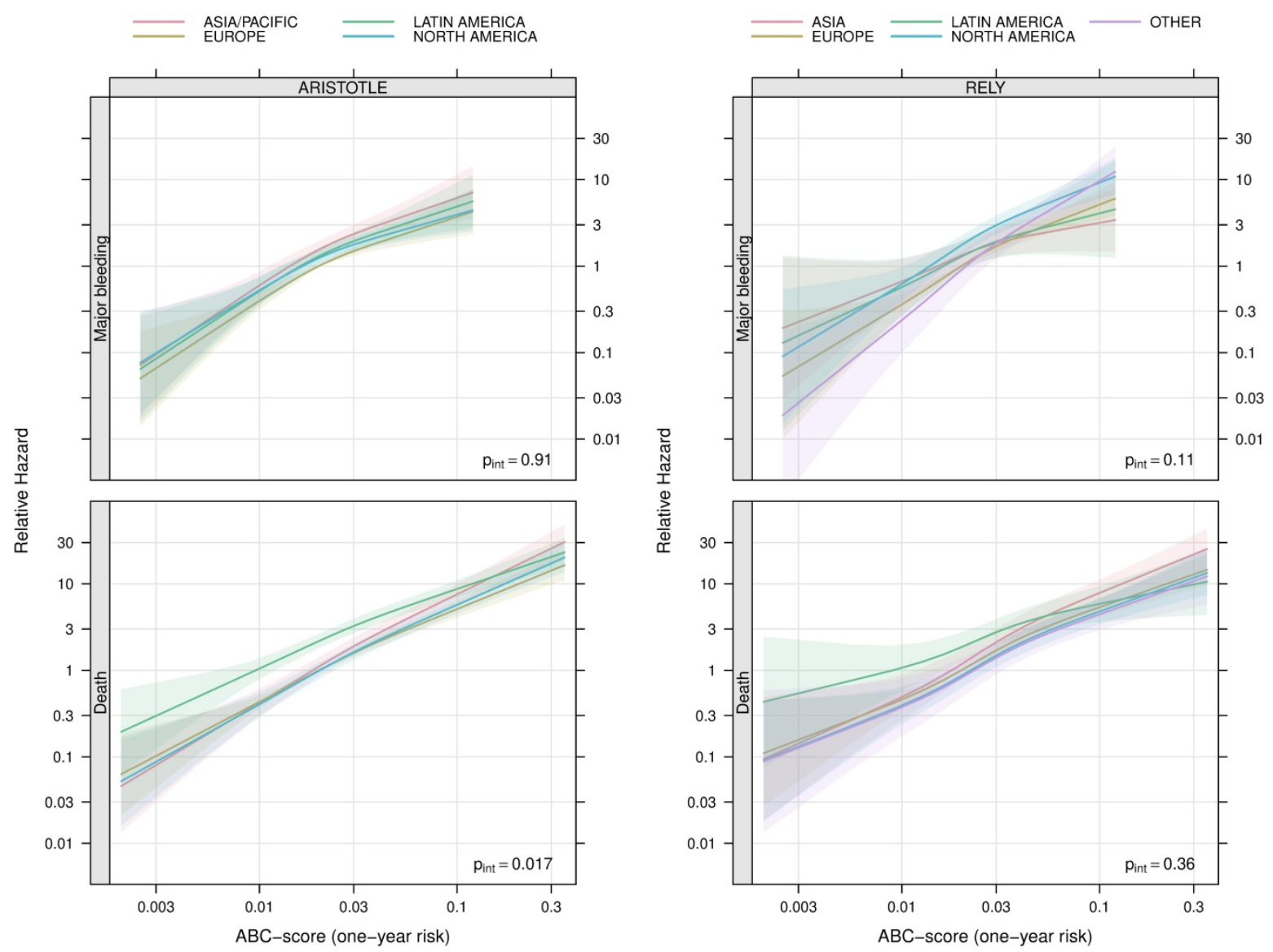

Figure 2 Relative hazard of major bleeding (top panels) and death (bottom panels) in relation to predicted ABC-AF-bleeding (top) and ABC-AF-death (bottom) 1-year risks among patients from different regions in the ARISTOTLE (left panels) and RE-LY (right panels) trials. An arbitrary reference point is set at a predicted ABC-AF-risk value of 0.02 in Europe. The $p$ value in each panel is for a test of no interaction between region and predicted ABC-AF-risk. ABC-AF bleeding score is age, biomarkers (troponin-hs, haemoglobin, and GDF-15 or renal function), clinical history (previous bleeding). ARISTOTLE, Apixaban for Reduction in Stroke and Other Thromboembolic Events in Atrial Fibrillation; RE-LY, Randomized Evaluation of Long-Term Anticoagulation Therapy.

levels, but another component might have been the lack of specific validation across geographic areas which is now eliminated. The findings from this study thus add novel information regarding the consistency of GDF-15 and the $\mathrm{ABC}-\mathrm{AF}-$-risk scores in $\mathrm{AF}$ and strengthen their role as a risk refinement tool applicable for a wide international usage.

\section{CONCLUSIONS}

The associations between GDF-15 and the biomarkerbased ABC-AF-bleeding and ABC-AF-death risk scores including GDF-15 and the outcomes major bleeding and death, in patients with $\mathrm{AF}$ on anticoagulation, are

Table 3 C-indices with 95\% Cl for the ABC-AF-bleeding risk score in ARISTOTLE and RE-LY comparing geographic regions

\begin{tabular}{|c|c|c|c|c|}
\hline & \multicolumn{2}{|c|}{ ARISTOTLE } & \multicolumn{2}{|l|}{ RE-LY } \\
\hline & C-index & $95 \% \mathrm{Cl}$ & C-index & $95 \% \mathrm{Cl}$ \\
\hline \multicolumn{5}{|l|}{ Major bleeding } \\
\hline All & 0.677 & 0.657 to 0.698 & 0.708 & 0.684 to 0.731 \\
\hline Asia/Pacific & 0.684 & 0.639 to 0.728 & 0.649 & 0.558 to 0.739 \\
\hline Europe & 0.680 & 0.641 to 0.719 & 0.701 & 0.664 to 0.739 \\
\hline Latin America & 0.674 & 0.629 to 0.719 & 0.657 & 0.561 to 0.754 \\
\hline North America & 0.659 & 0.620 to 0.699 & 0.710 & 0.672 to 0.749 \\
\hline Other & & & 0.760 & 0.698 to 0.822 \\
\hline
\end{tabular}

ABC-AF bleeding score, Age, Biomarkers (troponin T-hs, haemoglobin, and GDF-15), Clinical history (previous bleeding); ARISTOTLE, Apixaban for Reduction in Stroke and Other Thromboembolic Events in Atrial Fibrillation; RE-LY, Randomized Evaluation of Long-Term Anticoagulation Therapy. 
Table 4 C-indices with 95\% Cl for the ABC-AF-death risk score in ARISTOTLE and RE-LY comparing geographic regions.

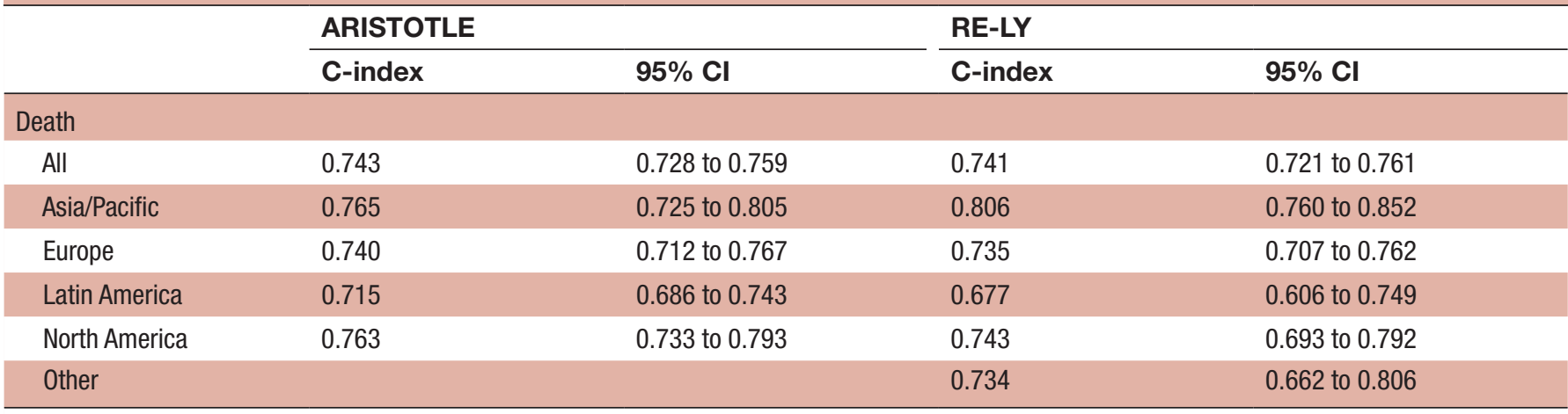

ABC-AF death score, Age, Biomarkers (NT-proBNP, troponin T-hs and GDF-15), Clinical history (heart failure); ARISTOTLE, Apixaban for Reduction in Stroke and Other Thromboembolic Events in Atrial Fibrillation; RE-LY, Randomized Evaluation of Long-Term Anticoagulation Therapy.

consistent across geographic regions. Accordingly, the discriminatory abilities of the ABC-AF-bleeding and ABCAF-death scores to prognosticate major bleeding and death are consistent and similarly clinically useful across global geographic regions. The assessment of geographic variation might be helpful as part of future validation processes when introducing new biomarkers or risk scores intended for a wide use.

\section{Author affiliations}

${ }^{1}$ Department of Medical Sciences, Cardiology, Uppsala University, Uppsala, Sweden

${ }^{2}$ Uppsala Clinical Research Center, Uppsala University, Uppsala, Sweden

${ }^{3}$ Duke Clinical Research Institute, Duke Health, Durham, North Carolina, USA

${ }^{4}$ INECO Neurociencias Oroño, Fundación INECO, Rosario, Argentina

${ }^{5}$ Gabriele d'Annunzio University, Chieti, Italy

${ }^{6}$ Population Health Res Inst, Hamilton, Ontario, Canada

${ }^{7}$ Thomas Jefferson Medical Coll and the Heart Ctr, Wynnewood, Pennsylvania, USA

${ }^{8}$ Mayo Clinic College of Medicine, Rochester, Minnesota, USA

${ }^{9}$ Boston Univ Medical Ctr, Boston, Massachusetts, USA

${ }^{10}$ Department of Medical Sciences, Clinical Chemistry, Uppsala University, Uppsala, Sweden

\section{Twitter M Cecilia Bahit @ceciliabahit}

Contributors TP, ZH, JL, AS and LW participated in the conception, design and interpretation of data. TP drafted the initial manuscript and all other authors contributed to revising it and have given final approval of the version to be submitted.

Funding The Apixaban for Reduction in Stroke and Other Thromboembolic Events in Atrial Fibrillation trial was funded by Bristol-Myers Squibb, Princeton, NJ, USA, and Pfizer, New York, NY, USA, and coordinated by the Duke Clinical Research Institute, Durham, NC, USA, and Uppsala Clinical Research Center, Uppsala, Sweden. The Randomized Evaluation of Long-Term Anticoagulation Therapy trial was funded by Boehringer Ingelheim, Ingelheim, Germany. The analyses were supported by Bristol-Myers Squibb and Pfizer, by grants from the Swedish Heart-Lung Foundation (20090183) and by grants from the Swedish Foundation for Strategic Research. Roche Diagnostics, Rotkreuz, Switzerland, supported the research by providing the growth differentiation factor 15 assay free of charge. The funding sources were given the opportunity to review and comment on the final version of the manuscript.

Competing interests TP reports institutional research grants from BristolMyers Squibb/Pfizer. ZH reports lecture and consulting fees from Boehringer Ingelheim, Bristol-Myers Squibb, Pfizer and Roche Diagnostics; consulting fees from Bristol-Myers Squibb, Meda, Merck Sharp \& Dohme, Pfizer and Roche Diagnostics and research grants from the Swedish Society for Medical Research (S17-0133) and the Swedish Heart-Lung Foundation (20170718). JL reports institutional research grants from Boehringer Ingelheim and Bristol-Myers Squibb/ Pfizer. JHA reports institutional research grants and consulting fees/honoraria from Bayer, Bristol-Myers Squibb, CryoLife and XaTek and consulting fees/ honoraria from Pfizer and Portola. MCB reports consulting fee/honoraria from Pfizer, MSD and CSL Behring. RDC reports fees, honoraria and research funding from Sanofi-Aventis, Amgen, Boehringer Ingelheim, Bayer, Bristol-Myers Squibb/ Pfizer, Daiichi Sankyo, Novartis, Portola and Roche. JWE reports institutional research grants and honoraria from Bayer, Boehringer Ingelheim, Bristol-Myers Squibb/Pfizer, Daiichi Sankyo, Janssen Pharmaceuticals, AstraZeneca, Eli Lilly, GlaxoSmithKline and Sanofi Aventis. MDE reports research grants and consulting fees from Pfizer, Bristol-Myers Squibb and Boehringer Ingelheim, consulting fees from Anthos Therapeutic, Alta Therapeutics and Boston Scientific. BJG has received other research support (DSMB) from Boston Scientific, Cardiovascular Research Foundation, Duke Clinical Research Institute, Duke University, Icahn School of Medicine at Mount Sinai, Janssen Research \& Development, Kowa Research Institute, Medtronic, Mount Sinai St. Luke's, Teva Pharmaceutical Industries and MyoKardia and consulting fees from Coretherapix SLU, Janssen Scientific Affairs and Sirtex Medical. CBG reports research grants and consulting/speaker fees from Boehringer Ingelheim, Bristol-Myers Squibb, Janssen Pharmaceuticals and Pfizer; research grants from AstraZeneca, Daichii Sankyo, AKROS, Apple, GlaxoSmithKline, Medtronic Foundation, Novartis Pharmaceutical Company and US Food \& Drug Administration and consulting/speaker fees from Bayer Corporation, USA, Boston Scientific Corporation, Abbvie, CeleCor Therapeutics, Correvio, Espero BioPharma, Medscape, Medtronic, Merck, National Institutes of Health, NovoNordisk, Roche Diagnostics and Rhoshan Pharmaceuticals. EMH reports research grant from Janssen; consulting/advisory board fees and honoraria from Boehringer Ingelheim; consulting/advisory board fees from Medtronic and Roche and honoraria from Bristol-Myers Squibb/Pfizer. RL reports institutional research grants and consulting fees from Bristol-Myers Squibb, Pfizer, GlaxoSmithKline, Medtronic PLC and Sanofi and consulting fees from Amgen, Bayer, Boehringer Ingelheim, Daiichi Sankyo, Merck and Portola. AS reports institutional research grants from AstraZeneca, Boehringer Ingelheim, Bristol-Myers Squibb/Pfizer, GlaxoSmithKline, Roche Diagnostics and consultancy fees from Olink Proteomics. LW reports institutional research grants from AstraZeneca, Boehringer Ingelheim, Bristol-Myers Squibb/ Pfizer, GlaxoSmithKline, Roche Diagnostics and Merck \& $\mathrm{C}$; consulting fees from Abbott and holds two patents involving growth differentiation factor 15 licensed to Roche Diagnostics (EP2047275B1 and US8951742B2).

Patient consent for publication Not required.

Ethics approval Approval by the appropriate ethics committees was obtained at all sites. All patients provided written informed consent.

Provenance and peer review Not commissioned; externally peer reviewed.

Data availability statement The data underlying this article will be shared on reasonable request to the corresponding author.

Open access This is an open access article distributed in accordance with the Creative Commons Attribution 4.0 Unported (CC BY 4.0) license, which permits others to copy, redistribute, remix, transform and build upon this work for any purpose, provided the original work is properly cited, a link to the licence is given and indication of whether changes were made. See: https://creativecommons.org/ licenses/by/4.0/

ORCID iDs

Tymon Pol http://orcid.org/0000-0001-6239-199X 
Johan Lindbäck http://orcid.org/0000-0002-6473-8798

Raffaele De Caterina http://orcid.org/0000-0003-1637-574X

\section{REFERENCES}

1 Hindricks G, Potpara T, Dagres N, et al. 2020 ESC guidelines for the diagnosis and management of atrial fibrillation developed in collaboration with the European association for Cardio-Thoracic surgery (EACTS). Eur Heart J 2021;42:373-498.

2 Hijazi Z, Aulin J, Andersson U, et al. Biomarkers of inflammation and risk of cardiovascular events in anticoagulated patients with atrial fibrillation. Heart 2016;102:508-17.

3 Hijazi Z, Wallentin L, Siegbahn A, et al. N-Terminal pro-B-type natriuretic peptide for risk assessment in patients with atria fibrillation: insights from the ARISTOTLE trial (apixaban for the prevention of stroke in subjects with atrial fibrillation). J Am Coll Cardiol 2013;61:2274-84

4 Aulin J, Siegbahn A, Hijazi Z, et al. Interleukin-6 and C-reactive protein and risk for death and cardiovascular events in patients with atrial fibrillation. Am Heart $J$ 2015;170:1151-60.

5 Hijazi Z, Oldgren J, Siegbahn A, et al. Biomarkers in atrial fibrillation: a clinical review. Eur Heart $J$ 2013;34:1475-80.

6 Wallentin L, Hijazi Z, Andersson U, et al. Growth differentiation factor 15, a marker of oxidative stress and inflammation, for risk assessment in patients with atrial fibrillation: insights from the apixaban for reduction in stroke and other thromboembolic events in atrial fibrillation (ARISTOTLE) trial. Circulation 2014:130:1847-58.

7 Hijazi Z, Oldgren J, Andersson U, et al. Growth-Differentiation factor 15 and risk of major bleeding in atrial fibrillation: insights from the randomized evaluation of long-term anticoagulation therapy (RE-LY) trial. Am Heart J 2017;190:94-103.

8 Hijazi Z, Oldgren J, Lindbäck J, et al. The novel biomarker-based $\mathrm{ABC}$ (age, biomarkers, clinical history)-bleeding risk score for patients with atrial fibrillation: a derivation and validation study. Lancet 2016;387:2302-11.

9 Hijazi Z, Oldgren J, Lindback J. A biomarker-based risk score to predict death in patients with atrial fibrillation: the $A B C$ (age, biomarkers, clinical history) death risk score. European heart journal 2017.

10 Goto S, Zhu J, Liu L, et al. Efficacy and safety of apixaban compared with warfarin for stroke prevention in patients with atrial fibrillation from East Asia: a subanalysis of the apixaban for reduction in stroke and other thromboembolic events in atrial fibrillation (ARISTOTLE) trial. Am Heart J 2014;168:303-9.

11 Hori M, Connolly SJ, Zhu J, et al. Dabigatran versus warfarin: effects on ischemic and hemorrhagic strokes and bleeding in Asians and non-Asians with atrial fibrillation. Stroke 2013;44:1891-6.

12 Wong KSL, Hu DY, Oomman A, et al. Rivaroxaban for stroke prevention in East Asian patients from the rocket AF trial. Stroke 2014;45:1739-47.

13 Yamashita T, Koretsune Y, Yang Y, et al. Edoxaban vs. Warfarin in East Asian Patients With Atrial Fibrillation - An ENGAGE AF-TIMI 48 Subanalysis. Circ J 2016;80:860-9.

14 Bahit MC, Granger CB, Alexander JH, et al. Regional variation in clinical characteristics and outcomes in patients with atrial fibrillation: findings from the ARISTOTLE trial. Int J Cardiol 2020;302:53-8.

15 Lopes RD, Alexander JH, Al-Khatib SM, et al. Apixaban for reduction in stroke and other thromboembolic events in atrial fibrillation (ARISTOTLE) trial: design and rationale. Am Heart J 2010;159:331-9.

16 Granger CB, Alexander JH, McMurray JJV, et al. Apixaban versus warfarin in patients with atrial fibrillation. $N$ Engl $J$ Med 2011;365:981-92.

17 Connolly SJ, Ezekowitz MD, Yusuf S, et al. Dabigatran versus warfarin in patients with atrial fibrillation. $N$ Engl J Med 2009;361:1139-51.

18 Ezekowitz MD, Connolly S, Parekh A, et al. Rationale and design of RE-LY: randomized evaluation of long-term anticoagulant therapy, warfarin, compared with dabigatran. Am Heart J 2009;157:805-10.

19 R Core Team. R: a language and environment for statistical computing, 2018R Foundation for Statistical Computing, Secondary R Core Team, Vienna, Austria. Available: https://www.R-project.org/

20 Frank E, Harrell J. Regression modeling strategies: with applications to linear models, logistic regression, and survival analysis. New York: Springer-Verlag, 2013. 